STRUCTURAL BIOLOGY

ISSN 2059-7983

Received 1 May 2019

Accepted 2 August 2019

Edited by Z. S. Derewenda, University of Virginia, USA

Keywords: direct electron transfer; flavin adenine dinucleotide-dependent dehydrogenase complex; glucose dehydrogenase; iron-sulfur cluster; hitchhiker protein; glucose sensors; Burkholderia cepacia.

PDB reference: $\gamma \alpha$-subunit complex from Burkholderia cepacia FAD glucose dehydrogenase, $6 \mathrm{a} 2 \mathrm{u}$

Supporting information: this article has supporting information at journals.iucr.org/d

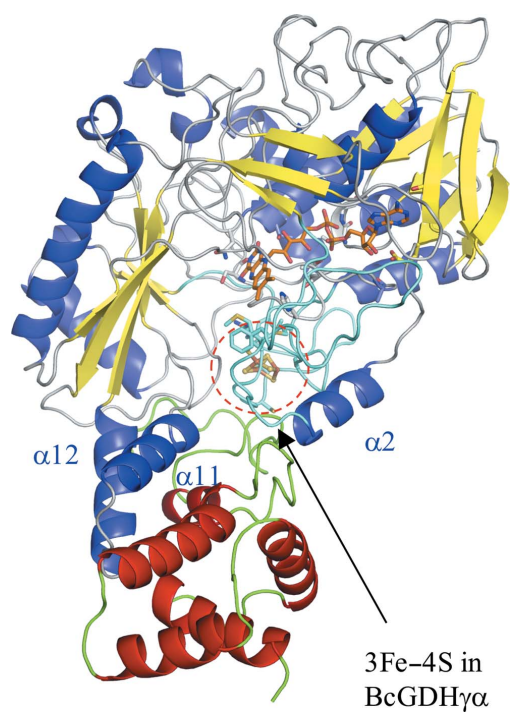

OPEN $\odot$ ACCESS

\section{X-ray structure of the direct electron transfer-type FAD glucose dehydrogenase catalytic subunit complexed with a hitchhiker protein}

\author{
Hiromi Yoshida, ${ }^{\text {a }}$ Katsuhiro Kojima, ${ }^{\text {b }}$ Masaki Shiota, ${ }^{\text {b }}$ Keiichi Yoshimatsu, ${ }^{c}$ \\ Tomohiko Yamazaki, ${ }^{\mathrm{d}}$ Stefano Ferri, ${ }^{\mathrm{e}}$ Wakako Tsugawa, ${ }^{\mathrm{b}}$ Shigehiro Kamitori ${ }^{\mathrm{a}}$ and \\ Koji Sode ${ }^{\text {b, f }}$ *
}

\begin{abstract}
a Life Science Research Center and Faculty of Medicine, Kagawa University, 1750-1 Ikenobe, Miki-cho, Kita-gun, Kagawa 761-0793, Japan, 'b Department of Biotechnology and Life Science, Graduate School of Engineering, Tokyo University of Agriculture and Technology, 2-24-16 Naka-cho, Koganei, Tokyo 184-8588, Japan, ${ }^{\mathbf{c} D e p a r t m e n t ~ o f ~}$ Chemistry, Missouri State University, Springfield, MO 65897, USA, ${ }^{\mathbf{d}}$ Research Center for Functional Materials, National Institute for Materials Science (NIMS), 1-2-1 Sengen, Tsukuba, Ibaraki 305-0047, Japan, ${ }^{\mathbf{e} D e p a r t m e n t ~ o f ~ A p p l i e d ~}$ Chemistry and Biochemical Engineering, Shizuoka University, 3-5-1 Johoku, Naka-ku, Hamamatsu, Shizuoka 432-8561, Japan, and fJoint Department of Biomedical Engineering, University of North Carolina at Chapel Hill and North Carolina State University, Chapel Hill, NC 27599, USA. *Correspondence e-mail: ksode@email.unc.edu
\end{abstract}

The bacterial flavin adenine dinucleotide (FAD)-dependent glucose dehydrogenase complex derived from Burkholderia cepacia (BcGDH) is a representative molecule of direct electron transfer-type FAD-dependent dehydrogenase complexes. In this study, the X-ray structure of $\mathrm{BcGDH} \gamma \alpha$, the catalytic subunit ( $\alpha$-subunit) of BcGDH complexed with a hitchhiker protein ( $\gamma$-subunit), was determined. The most prominent feature of this enzyme is the presence of the $3 \mathrm{Fe}-4 \mathrm{~S}$ cluster, which is located at the surface of the catalytic subunit and functions in intramolecular and intermolecular electron transfer from FAD to the electron-transfer subunit. The structure of the complex revealed that these two molecules are connected through disulfide bonds and hydrophobic interactions, and that the formation of disulfide bonds is required to stabilize the catalytic subunit. The structure of the complex revealed the putative position of the electron-transfer subunit. A comparison of the structures of BcGDH $\gamma \alpha$ and membrane-bound fumarate reductases suggested that the whole BcGDH complex, which also includes the membrane-bound $\beta$-subunit containing three heme $c$ moieties, may form a similar overall structure to fumarate reductases, thus accomplishing effective electron transfer.

\section{Introduction}

Various sugar oxidoreductases (dehydrogenases) have been reported to be inherently capable of direct electron transfer to electrodes composed of carbon materials or to gold electrodes. These dehydrogenases harbor an electron-transfer domain or subunit, together with a catalytic domain or subunit. The catalytic domains or subunits, which are responsible for catalyzing sugar oxidation, are categorized by their cofactor: flavin adenine dinucleotide (FAD) or pyrroloquinoline quinone (PQQ). The electron-transfer domains or subunits, which are responsible for transferring electrons to the external electron acceptor, are also categorized by the type of heme (heme $b$ or $c$ ) that is present in the electron-transfer domain or subunit.

One of the representative groups of direct electron transfertype (DET-type) dehydrogenases consists of cellobiose dehydrogenases (CDHs), which are composed of a catalytic domain harboring FAD and a heme $b$-type electron-transfer domain. In CDHs, open and closed states were identified by 
the approach of the cytochrome domain to the catalytic domain via a flexible linker (Tan et al., 2015).

The other representative protein group that is capable of direct electron transfer consists of FAD-dependent dehydrogenase complexes. These complexes are composed of a catalytic subunit with FAD, an electron-transfer subunit containing three heme $c$ moieties and a small subunit. The isolation, characterization, bioelectrochemical studies and application of several bacterial FAD-dependent dehydrogenase complexes have been reported, including bacterial glucose dehydrogenase (FADGDH; Inose et al., 2003; Sode et al., 1996; Tsuya et al., 2006; Yamazaki et al., 1999; Yamaoka \& Sode, 2007; Yamaoka et al., 2008), fructose dehydrogenase (FDH; Ameyama et al., 1981; Kawai et al., 2013;), 2-ketoD-gluconate dehydrogenase (KGDH; Kataoka et al., 2015; Shinagawa et al., 1981) and sorbitol dehydrogenase (Toyama et al., 2005). These FAD-dependent dehydrogenase complexes have the potential to directly transfer electrons to an electrode because of the presence of the heme $c$ subunit. However, no structural information is currently available for any subunits from DET-type FAD-dependent dehydrogenase complexes.

Our research group has been studying a representative FAD-dependent dehydrogenase complex (FADGDH) derived from Burkholderia cepacia SM4 (BcGDH). BcGDH comprises three distinct subunits: the catalytic subunit ( $\alpha$-subunit), which contains an FAD cofactor in its redox center, shows catalytic activity and oxidizes the first hydroxyl group of glucose, the small subunit ( $\gamma$-subunit), a hitchhiker protein of the bacterial TAT secretion system that is necessary for the proper folding and secretion of the $\alpha$-subunit (Yamaoka et al., 2004), and the membrane-bound subunit with three heme $c$ moieties ( $\beta$-subunit) that is responsible for the transfer of electrons between the active-site cofactor and external electron acceptors. Owing to the presence of the $\beta$-subunit, $\mathrm{BcGDH}$ is capable of transferring electrons directly to an electrode, making it an ideal molecule for glucose sensors and applications in a variety of biomedical devices (Sode et al., 2016; Yamashita et al., 2018). In addition, the $\mathrm{BcGDH} \gamma \alpha$ complex, which is $\mathrm{BcGDH}$ lacking the $\beta$-subunit, also exhibits dye-mediated glucose dehydrogenase activity (Inose et al., 2003). Recently, based on biochemical analyses and electron paramagnetic resonance spectroscopy, we reported the presence of a $3 \mathrm{Fe}-4 \mathrm{~S}$ cluster in the catalytic subunit (Shiota et al., 2016). The 3Fe-4S cluster is located in the cysteine-rich region, which is conserved in the catalytic subunits of previously reported FAD-dependent dehydrogenase complexes. The $3 \mathrm{Fe}-4 \mathrm{~S}$ cluster is responsible for electron transfer from FAD (intramolecular) to the multiheme $c$ subunit (intermolecular), which is the key position for understanding the features of this group of enzymes that are capable of direct electron transfer.

Another notable feature of the enzymes in the FADdependent dehydrogenase complex is the presence of a small subunit that is essential for the functional expression of the FAD-harboring catalytic subunit. Considering their primary structure, particularly the signal sequences necessary for secretion, these small subunits are predicted to be hitchhiker proteins that are needed for secretion of the catalytic subunit into the periplasmic space (Yamaoka et al., 2004). However, no structural information is available for any types of hitchhiker proteins or their complexes with targeted proteins.

In this study, we determined the X-ray structure of $\mathrm{BcGDH} \gamma \alpha$, consisting of the BcGDH catalytic subunit complexed with the small (hitchhiker) subunit. The structure of the FAD-binding catalytic subunit was similar to those of several glucose-methanol-choline (GMC) oxidoreductases. The catalytic sites of $\mathrm{BcGDH} \gamma \alpha$ were conserved compared with other GMC oxidoreductases. In the structure of $\mathrm{BcGDH} \gamma \alpha$, the $3 \mathrm{Fe}-4 \mathrm{~S}$ cluster was located at the surface of the catalytic subunit. The structure of the complex of the catalytic subunit with the small subunit revealed that these two molecules were connected through disulfide bonds and hydrophobic interactions. Site-directed mutagenesis studies were performed to elucidate the role of the disulfide bond. The structural similarities to other FAD-dependent dehydrogenase complexes and to fumarate reductase are also discussed.

\section{Materials and methods}

\subsection{Recombinant expression of BcGDH $\gamma \alpha$}

The structural genes encoding the catalytic $(\alpha)$ and small $(\gamma)$ GDH subunits of B. cepacia sp. SM4 (FERMBP-7306) were subcloned into the high-expression vector pTrc99A with a His tag at the C-terminus of the $\alpha$-subunit. The constructed plasmid (designated pTrc $\gamma \alpha$-His) was transformed into the bacterial host Escherichia coli BL21 (DE3) for expression. In the present study, a complex of the $\gamma$-subunit and His-tagged $\alpha$ subunit was expressed as recombinant wild-type $\mathrm{BcGDH} \gamma \alpha$. The $\gamma$-subunit consists of 168 amino acids $(18 \mathrm{kDa})$, including 47 amino acids of the signal peptide at the $\mathrm{N}$-terminal region, and the mature $\gamma$-subunit contains 121 amino acids (13 kDa). The $\alpha$ subunit consists of 539 amino acids and was produced as a catalytic domain of $60 \mathrm{kDa}$.

Transformed E. coli were cultured in $500 \mathrm{ml}$ conical flasks containing $100 \mathrm{ml} \mathrm{ZYP-5052} \mathrm{medium} \mathrm{(Studier,} \mathrm{2005)} \mathrm{in} \mathrm{a}$ rotary shaker at $293 \mathrm{~K}$ for $48 \mathrm{~h}$.

The E. coli selenium auxotroph strain B834 (DE3) was used to produce selenomethionine-containing $\mathrm{BcGDH} \gamma \alpha$. The E. coli B834 (DE3) cells harboring $\mathrm{p} \operatorname{Trc} \gamma \alpha$-His were cultured in $500 \mathrm{ml}$ conical flasks containing $100 \mathrm{ml}$ PASM-5052 medium (Studier, 2005) in a rotary shaker at $293 \mathrm{~K}$ for $211 \mathrm{~h}$.

The cells were harvested by centrifugation and then resuspended in $20 \mathrm{~m} M$ sodium phosphate buffer containing $20 \mathrm{~m} M$ imidazole and $0.5 \mathrm{M} \mathrm{NaCl} \mathrm{pH} \mathrm{7.0.} \mathrm{After} \mathrm{resuspension,} \mathrm{the}$ cells were disrupted with a French press. The lysate was centrifuged at $10000 \mathrm{~g}$ for $15 \mathrm{~min}$ at $277 \mathrm{~K}$ to remove the the insoluble fraction consisting of cell debris and inclusion bodies. The resulting supernatant, which was designated the crude extract, was purified by FPLC.

\subsection{Enzyme purification}

The recombinant BcGDH $\gamma \alpha$ complex was purified using nickel-chelate chromatography and cation-exchange 
chromatography. The crude extract was loaded onto a HisTrap HP column (1 ml; GE Healthcare Life Sciences, Uppsala, Sweden) that had been equilibrated with $20 \mathrm{~m} M$ sodium phosphate buffer containing $20 \mathrm{~m} M$ imidazole and $0.5 \mathrm{M} \mathrm{NaCl}$ $\mathrm{pH} 7.0$ and was washed with the same buffer. GDH was then eluted with ten column volumes of a stepwise imidazole gradient (70, 380 and $500 \mathrm{~m} M$ imidazole in $20 \mathrm{~m} M$ sodium phosphate buffer and $0.5 \mathrm{M} \mathrm{NaCl} \mathrm{pH} \mathrm{7.0)} \mathrm{at} \mathrm{a} \mathrm{rate} \mathrm{of}$ $1 \mathrm{ml} \mathrm{min}{ }^{-1}$ for each step. The fractions with the highest activities were pooled and dialyzed overnight in $10 \mathrm{mM}$ potassium phosphate buffer $\mathrm{pH}$ 6.0.

The pooled fractions were subsequently loaded onto a Resource S column (5 ml; GE Healthcare, Little Chalfont, England) that had been equilibrated with $10 \mathrm{~m} M$ potassium phosphate buffer $\mathrm{pH} 6.0$ and were washed with the same buffer. GDH was eluted with 20 column volumes of a linear $\mathrm{NaCl}$ gradient $(0-1 M \mathrm{NaCl}$ in $10 \mathrm{~m} M$ potassium phosphate buffer $\mathrm{pH} 6.0$ ) at a rate of $5 \mathrm{ml} \mathrm{min}^{-1}$. The purified enzyme was concentrated to $8.9 \mathrm{mg} \mathrm{ml}^{-1}$ and the buffer was exchanged to Milli-Q water using Amicon Ultra-15 (nominal molecular-weight limit 3000; Merck Millipore, Carrigtwohill, Ireland). The protein concentrations were measured using a DC Protein Assay kit (Bio-Rad Laboratories, Hercules, California, USA).

\subsection{Site-directed mutagenesis}

Site-directed mutagenesis of the target amino acids (Cys213 in the $\alpha$-subunit and Cys152 in the $\gamma$-subunit) was accomplished using the QuikChange mutagenesis kit (Agilent, Santa Clara, California, USA) according to the manufacturer's instructions. All mutations were confirmed by nucleotide sequencing.

\subsection{Enzyme assay}

The activities of crude extracts and the purified recombinant $\mathrm{BcGDH} \gamma \alpha$ complex were determined using methods described in a previous study (Inose et al., 2003) with slight modifications. The enzyme sample was incubated at room temperature with $10 \mathrm{~m} M$ potassium phosphate buffer $\mathrm{pH} 7.0$ containing $6 \mathrm{mM}$ 5-methylphenazinium methylsulfate (phenazine methosulfate; PMS), $0.06 \mathrm{mM}$ 2,6-dichlorophenolindophenol (DCIP) and various concentrations of glucose. The activity was determined by monitoring the decrease in the absorbance of DCIP at $600 \mathrm{~nm}$ and using the molar absorption coefficient of DCIP (16.3 mM cm$~^{-1}$ at $\left.\mathrm{pH} 7.0\right)$ to calculate the enzyme activity. The molar absorption coefficient of DCIP was determined by measuring the absorbance of fixed concentrations of DCIP at $600 \mathrm{~nm}$ in $10 \mathrm{~m} M$ potassium phosphate buffer $\mathrm{pH}$ 7.0. One unit of enzyme activity is defined as the amount of enzyme that oxidizes $1 \mu \mathrm{mol}$ glucose per minute.

\subsection{Crystallization}

Initial crystal screening was performed using the sittingdrop vapor-diffusion method with a Mosquito system (TTP Labtech, Hertfordshire, England). The protein concentration of wild-type $\mathrm{BcGDH} \gamma \alpha$ was $8.9 \mathrm{mg} \mathrm{ml}^{-1}$ in Milli-Q water.
After a few days, yellow crystals were observed in a reservoir solution containing $60 \%$ Tacsimate $\mathrm{pH}$ 7.0. Well diffracting crystals were obtained in a droplet containing a mixture of $1.5 \mu \mathrm{l}$ protein solution $\left(5.7 \mathrm{mg} \mathrm{ml}^{-1}\right.$ in Milli-Q water) and $0.75 \mu \mathrm{l}$ reservoir solution (59.9-60.2\% Tacsimate $\mathrm{pH}$ 7.0) in a well containing $50 \mu \mathrm{l}$ reservoir solution using the sitting-drop method at $293 \mathrm{~K}$.

The methionines in $\mathrm{BcGDH} \gamma \alpha$ were replaced with selenomethionines (SeMet BcGDH $\gamma \alpha$ ) in order to determine the initial phases for the structure factors of wild-type $\mathrm{BcGDH} \gamma \alpha$. However, crystals of SeMet BcGDH $\gamma \alpha$ were not obtained under the same conditions as those of wild-type $\mathrm{BcGDH} \gamma \alpha$. Since the prepared SeMet BcGDH $\gamma \alpha$ contained a small amount of nonprocessed $\gamma$-subunit $(18 \mathrm{kDa})$, which was observed on SDS-PAGE gels, the crystallization of SeMet $\mathrm{BcGDH} \gamma \alpha$ was attempted in the presence of proteases using Proti-Ace (Hampton Research, California, USA). Some crystals of SeMet BcGDH $\gamma \alpha$ appeared in a droplet consisting of $1.0 \mu \mathrm{l}$ protein solution ( $10.2 \mathrm{mg} \mathrm{ml}^{-1}$ in Milli-Q water), $0.2 \mu \mathrm{l}$ of a $0.1 \mathrm{mg} \mathrm{ml}^{-1}$ subtilisin solution and $1.0 \mu \mathrm{l}$ reservoir solution (60\% Tacsimate $\mathrm{pH} 7.0)$ in a well containing $50 \mu \mathrm{l}$ reservoir solution using the sitting-drop method at $293 \mathrm{~K}$.

\subsection{X-ray crystallography}

Single crystals of both wild-type $\mathrm{BcGDH} \gamma \alpha$ and SeMet $\mathrm{BcGDH} \gamma \alpha$ were mounted in cryoloops and directly flashcooled in a stream of nitrogen gas at $100 \mathrm{~K}$. X-ray diffraction data were collected using an ADSC Quantum 270 CCD detector system on the PF-AR NE3A beamline at the High Energy Accelerator Research Organization (KEK), Tsukuba, Japan. Diffraction data were processed using $H K L-2000$ (Otwinowski \& Minor, 1997) and the CCP4 suite (Winn et al., 2011). Although X-ray diffraction data were collected from the crystal of BcGDH $\gamma \alpha$ to $2.2 \AA$ resolution, the data actually used for structure determination were truncated at $2.6 \AA$ resolution owing to an extensively high $R_{\text {merge }}$ in the outermost shell. The unit-cell parameters of the crystal were large ( $a=b=110.5, c=524.9 \AA)$ and the diffraction pattern was characterized by strong anisotropy.

The initial phases of SeMet BcGDH $\gamma \alpha$ were obtained using the single-wavelength anomalous dispersion (SAD) method with the AutoSol program (Terwilliger, 2004). Since the unitcell parameters of the crystal of wild-type BcGDH $\gamma \alpha$ were isomorphous to those of the SeMet derivative, the phases were transferred directly to the former and the model was constructed using AutoBuild in the PHENIX system (Adams et al., 2010; Afonine et al., 2012).

Further model building and structure refinement were performed using Coot (Emsley et al., 2010) and REFMAC5 (Murshudov et al., 2011), respectively. The structure was validated using PROCHECK (Laskowski et al., 1993, 2001).

The anomalous dispersion of $\mathrm{Fe}$ atoms was utilized in order to determine the number and the positions of $\mathrm{Fe}$ atoms in the iron-sulfur cluster. A SAD data set from a crystal of wild-type BcGDH $\gamma \alpha$ was collected to $1.74086 \AA$ resolution on beamline PF-AR NW12A at KEK using an ADSC Quantum 210r CCD detector system. 
Table 1

Data-collection and refinement statistics.

Values in parentheses are for the highest resolution bin.

\begin{tabular}{|c|c|c|c|}
\hline & $\mathrm{BcGDH} \gamma \alpha$ & $\mathrm{BcGDH} \gamma \alpha$ & SeMet BcGDH $\gamma \alpha$ \\
\hline \multicolumn{4}{|l|}{ Data collection } \\
\hline Beamline & PF-AR NE3A & PF NW12A & PF-AR NE3A \\
\hline Temperature (K) & 100 & 100 & 100 \\
\hline Wavelength $(\AA)$ & 1.0 & 1.74086 & 0.97892 \\
\hline Resolution range $(\AA)$ & $50.0-2.60(2.64-2.60)$ & $50.0-2.90(2.95-2.90)$ & $50.0-3.40(3.46-3.40)$ \\
\hline No. of measured reflections & 1273970 & 369135 & 929441 \\
\hline No. of unique reflections & 60005 & 43770 & 49132 \\
\hline Multiplicity & $21.2(21.4)$ & $4.9(6.8)$ & $18.9(19.0)$ \\
\hline Completeness (\%) & $99.9(100.0)$ & $96.7(99.9)$ & $100.0(100.0)$ \\
\hline Mean $I / \sigma(I)$ & $24.4(10.0)$ & $20.2(5.6)$ & $43.1(12.6)$ \\
\hline$R_{\text {merge }} \dagger(\%)$ & $15.0(42.8)$ & $13.5(46.9)$ & $16.2(48.5)$ \\
\hline Space group & $P 6_{5} 22$ & $P 6_{5} 22$ & $P 6_{5} 22$ \\
\hline$a, b, c(\AA)$ & $110.52,110.52,524.88$ & $110.72,110.72,525.42$ & $110.48,110.48,524.09$ \\
\hline$\alpha, \beta, \gamma\left({ }^{\circ}\right)$ & $90,90,120$ & $90,90,120$ & $90,90,120$ \\
\hline \multicolumn{4}{|l|}{ Refinement } \\
\hline Resolution range $(\AA)$ & $43.74-2.60(2.67-2.60)$ & & \\
\hline No. of reflections & $56812(4078)$ & & \\
\hline Completeness (\%) & $99.9(99.2)$ & & \\
\hline$R$ factor $(\%)$ & $20.5(27.3)$ & & \\
\hline$R_{\text {free }}(\%)$ & $26.1(37.6)$ & & \\
\hline R.m.s.d., bond lengths $(\AA)$ & 0.003 & & \\
\hline R.m.s.d., bond angles $\left({ }^{\circ}\right)$ & 0.6 & & \\
\hline \multicolumn{4}{|l|}{ Ramachandran plot } \\
\hline Most favored region (\%) & 84.6 & & \\
\hline Additional allowed region (\%) & 15.0 & & \\
\hline \multicolumn{4}{|c|}{$B$ factors $\left(\AA^{2}\right)$} \\
\hline Protein & 45.6 & & \\
\hline Cofactor FAD & 31.6 [two molecules] & & \\
\hline $3 \mathrm{Fe}-4 \mathrm{~S}$ & 33.0 [two molecules] & & \\
\hline Water & $29.9^{2}$ & & \\
\hline PDB code & $6 \mathrm{a} 2 \mathrm{u}$ & & \\
\hline
\end{tabular}

$\dagger R_{\text {merge }}=\sum_{h k l} \sum_{i}\left|I_{i}(h k l)-\langle I(h k l)\rangle\right| / \sum_{h k l} \sum_{i} I_{i}(h k l)$, where $I_{i}(h k l)$ is the $i$ th measurement and $\langle I(h k l)\rangle$ is the weighted mean of all measurements of $I(h k l)$.

Data-collection and refinement statistics for all data sets are listed in Table 1. Figs. 1, 2, 3, 4, 6 and Supplementary Figs. S2, S5, S6, S7 and S9 were generated using PyMOL (Schrödinger, New York, USA).

\section{Results}

\subsection{The overall structure of $\mathrm{BcGDH} \gamma \alpha$}

Fig. 1 and Supplementary Fig. S1 show the overall structure of the complex of the $\gamma$ - and $\alpha$-subunits of BcGDH and the topology of the protein, respectively. The $\gamma$-subunit consists of five $\alpha$-helices (colored red). The overall structure of the $\alpha$-subunit comprises $15 \alpha$-helices (colored blue) and 17 $\beta$-strands and adopts an FAD-binding fold. The additional domain contains a six-stranded antiparallel $\beta$-sheet surrounded by six $\alpha$-helices and a protruding loop including two $\alpha$-helices ( $\alpha 11$ and $\alpha 12)$ facing towards the $\gamma$-subunit. Two distinguishing long loop regions are located between $\beta 2$ and $\beta 3$ and between $\beta 4$ and $\alpha 8$. The former loop (Ala39-Leu83) contains a unique $\alpha$-helix $(\alpha 2)$ that is located in close proximity to the $\gamma$-subunit at the center of the $\gamma \alpha$-subunit complex and above the iron-sulfur cluster shown in the red circle [Fig. 1(a)], which is described later. The latter loop (Glu197Asn229) surrounds the iron-sulfur cluster and contains a cysteine cluster (Cys212, Cys213, Cys218 and Cys222); three of the four cysteines are involved in forming the $3 \mathrm{Fe}-4 \mathrm{~S}$ cluster.

The structure around FAD in the $\alpha$-subunit is shown in Fig. 2. FAD is surrounded by part of the long loop between Ala98 and Ser109, $\alpha 1, \alpha 15, \beta 2$ and $\beta 6$. Since continuous electron density was observed between FAD and His105 in the $\alpha$-subunit of $\mathrm{BcGDH} \gamma \alpha$ [Fig. 2(b)], a covalent bond was deemed to form between FAD and His105 in the $\alpha$-subunit of BcGDH.

In the crystal, two pairs of $\mathrm{BcGDH} \gamma \alpha$ complexes were observed in the asymmetric unit (Supplementary Fig. S2). As shown in the surface model presented in Figs. 1(a) and 1(b), BcGDH $\gamma \alpha$ forms a heterodimer with a bent form, and the bent complex ( $\gamma \alpha$-subunits) faces the back side of the other complex in the crystal (Supplementary Fig. S2). Although the protein seems to form a heterotetramer, $\gamma \alpha \gamma^{\prime} \alpha^{\prime}$, according to PISA analysis (http://www.ebi.ac.uk/ $\mathrm{pdbe} / \mathrm{pisa} /$ ) this assembly is unstable and forms two pairs of $\gamma$ - and $\alpha$-subunits $\left(\gamma \alpha\right.$ and $\left.\gamma^{\prime} \alpha^{\prime}\right)$. A gel-filtration chromatogram revealed the absence of an oligomeric form of $\mathrm{BcGDH} \gamma \alpha$ in solution (data not shown). Therefore, the observed $\gamma \alpha \gamma^{\prime} \alpha^{\prime}$ heterotetramer in the crystal structure is an artifact of crystallization. Since each molecule of $\gamma \alpha$ and $\gamma^{\prime} \alpha^{\prime}$ is almost identical, with r.m.s. deviations for $\mathrm{C}^{\alpha}$ atoms of 0.48 ( $\alpha$ and $\alpha^{\prime}$ ) and 0.89 ( $\gamma$ and $\gamma^{\prime}$ ), the structural description concentrates on $\gamma \alpha$ unless otherwise specified.

\subsection{Iron-sulfur cluster}

The locations of Fe atoms were identified using the anomalous dispersion method and were observed in the expected positions of the iron-sulfur cluster. Three Fe-atom sites were identified in the iron-sulfur cluster of each molecule [Figs. 3(a)-3(d)]. The simulated-annealing OMIT maps of sulfur ions in the iron-sulfur cluster and the disulfide bond indicated that this iron-sulfur cluster is a $3 \mathrm{Fe}-4 \mathrm{~S}$ cluster coordinated by Cys212, Cys218 and Cys222 of the $\alpha$-subunit. A neighboring cysteine, Cys213, in the $\alpha$-subunit forms a disulfide bond with Cys152 of the $\gamma$-subunit. The unique cysteine cluster of $\mathrm{BcGDH} \gamma \alpha$ contributes to the formation of the $3 \mathrm{Fe}-4 \mathrm{~S}$ cluster for electron transfer and the disulfide bond for stabilization of the structure of the $\gamma \alpha$ complex.

\subsection{Interface between the $\gamma$ - and $\alpha$-subunits}

The $\gamma$-subunit tightly binds the $\alpha$-subunit and forms a stable heterodimer. The hydrophobic loop region at the C-terminus 
of the $\gamma$-subunit (colored green in the black circle in Fig. 4) contacts one of the distinguishing long loops, including $\alpha 2$ in the $\alpha$-subunit at the interface (colored in cyan). A disulfide bond is located at the interface between the $\gamma$ - and $\alpha$-subunits (Cys152 in the $\gamma$-subunt and Cys213 in the $\alpha$-subunit), as indicated by the red circle in Fig. 4, and is in close proximity to the hydrophobic cluster created by the C-terminal region in the $\gamma$-subunit indicated by the black circle (Leu146, Val147, Ile148, Pro153, Pro156, Gly157, Phe158, Trp159, Ala160 and Pro163) and the surrounding residues of the $\alpha$-subunit (Leu172, Pro173, Leu174, Phe176, Leu333, Trp334, Pro335, Gly336, Gly338, Pro339 and Met342). A $3 \mathrm{Fe}-4 \mathrm{~S}$ cluster is located next to the disulfide bond, and the iron-sulfur cluster is located in the hydrophobic environment formed by the above hydrophobic cluster, the hydrophobic loop region (Met219, Pro223, Ile224, Ala226 and Met227, colored cyan) of the $\alpha$-subunit, including four cysteine residues (Cys212, Cys213, Cys218 and Cys222) involved in formation of the $3 \mathrm{Fe}-4 \mathrm{~S}$ cluster and the disulfide bond, and two alanine residues (Ala107 and Ala108) located between 3Fe-4S and the isoalloxazine ring of FAD. Furthermore, $\alpha 4$ and $\alpha 5$ of the $\gamma$-subunit make contacts with protruding helices, including the $\alpha 11$ and $\alpha 12$ helices of the $\alpha$-subunit (in the area indicated by the blue circle). These hydrophobic contacts contribute to the tightly bound subunit interface.

\subsection{Site-directed mutagenesis}

The crystal structure of the $\mathrm{BcGDH} \gamma \alpha$ complex revealed the presence of an inter-subunit disulfide bond between the side chains of Cys213 in the $\alpha$-subunit and Cys152 in the $\gamma$-subunit. As reported previously (Shiota et al., 2016), substitution of Cys213 in the $\alpha$-subunit by serine $[\gamma \alpha$ (Cys213Ser) $]$ only exerts a limited effect on the kinetic parameters of the $\mathrm{BcGDH} \gamma \alpha$ complex at room temperature. Thus, the inter-subunit disulfide bond is not essential for enzyme activity. A mutant $\mathrm{BcGDH} \gamma \alpha$ complex with a Cys152Ser mutation in the $\gamma$-subunit $[\gamma($ Cys152Ser $) \alpha]$ was constructed and characterized to further confirm this hypothesis. Table 2 presents the kinetic parameters of the


(a)



(b)

Figure 1

Overall structure of $\mathrm{BcGDH} \gamma \alpha$. (a) Overall structure of $\mathrm{BcGDH} \gamma \alpha$ shown as cartoon (left) and surface (right) models. In the cartoon model, the $\gamma$-subunit is shown as red $\alpha$-helices and green loop regions, and the $\alpha$-subunit is represented with blue $\alpha$-helices, yellow $\beta$-strands and gray loop regions. In the surface model, the $\gamma$ - and $\alpha$-subunits are shown in red and blue, respectively, and are tightly bound to each other. The bound FAD is represented as an orange stick model. The cysteine cluster indicated with a red circle includes the iron-sulfur cluster shown as spheres. $(b)$ A view of the model rotated $180^{\circ}$ around the $x$ axis. 
mutant $\mathrm{BcGDH} \gamma \alpha$ complex at room temperature. In the absence of the electron-transfer subunit ( $\beta$-subunit), the $\mathrm{BcGDH} \gamma \alpha$ complex shows relatively low dye-mediated glucose dehydrogenase activity at the conventionally used concentration of the primary electron accepter (PMS; Yamazaki et al., 1999). Therefore, the enzyme activity of the BcGDH $\gamma \alpha$ complex was determined using $6 \mathrm{~m} M$ PMS, a concentration that is tenfold higher than the condition used for the $\mathrm{BcGDH}$ complex containing the electron-transfer subunit (Supplementary Fig. S4). The $V_{\max }$ of the $\gamma($ Cys152Ser $) \alpha$ complex is only moderately lower than that of the wild-type $\gamma \alpha$ complex. The $K_{\mathrm{m}}$ of the $\gamma($ Cys152Ser $) \alpha$ complex is comparable to the $K_{\mathrm{m}}$ of the wild-type $\gamma \alpha$ complex.

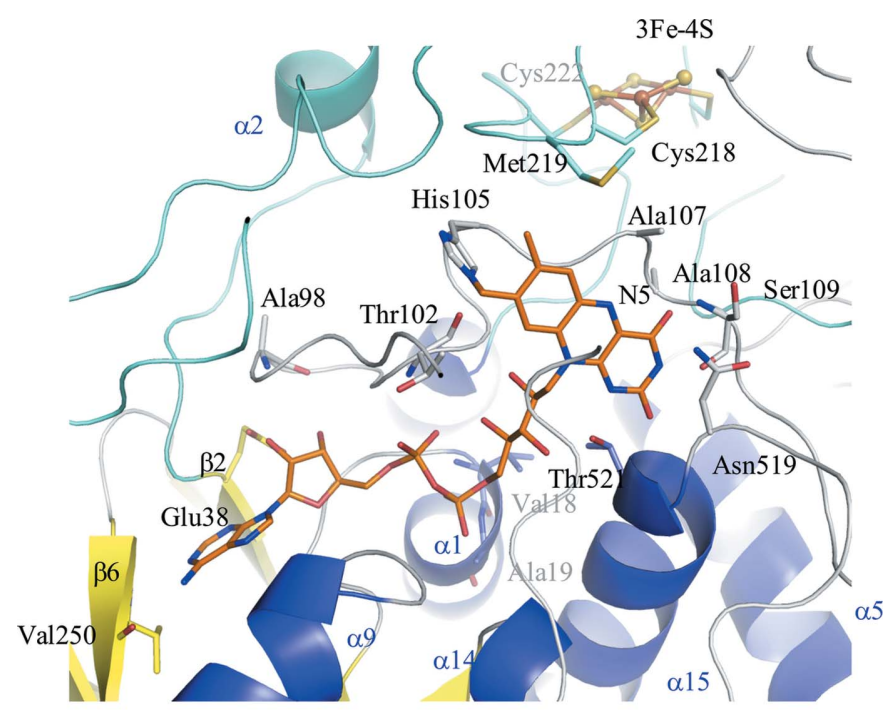

(a)

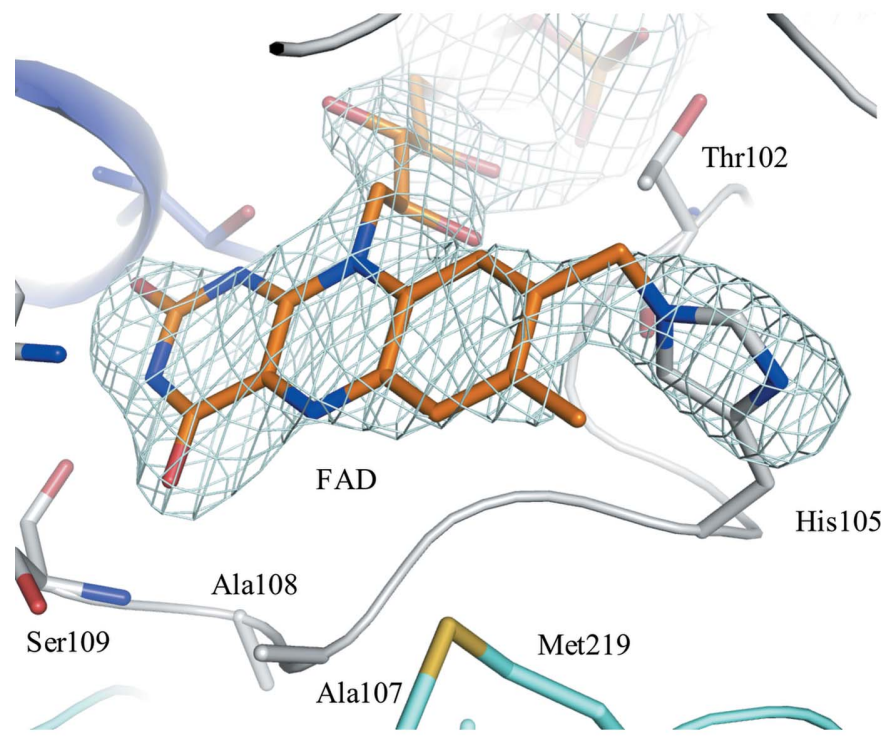

(b)

Figure 2

FAD-binding site and FAD covalently bound to His105 of the $\alpha$-subunit. (a) FAD-binding site with the surrounding environment. A long loop of $\alpha$-subunit including $\alpha 2$ is colored cyan. FAD is shown as an orange stick model. Other colors are the same as in Fig. 1. (b) The simulated-annealing OMIT maps of FAD and His105 in wild-type GDH $\gamma \alpha$ contoured at $4 \sigma$ are shown in light blue. FAD is shown as an orange stick model.
Table 2

Kinetic parameters of $\mathrm{BcGDH} \gamma \alpha$ mutants measured at room temperature.

Plots of the measurements from which the values were calculated are shown in Supplementary Fig. S3.

\begin{tabular}{llc}
\hline Sample & $K_{\mathrm{m}}(\mathrm{m} M)$ & $V_{\max }\left(\mathrm{U} \mathrm{mg}^{-1}\right)$ \\
\hline Wild type & $0.97 \pm 0.052$ & $120 \pm 1.5$ \\
$\gamma \alpha(\mathrm{C} 213 \mathrm{~S})$ & $0.76 \pm 0.036$ & $76 \pm 0.78$ \\
$\gamma(\mathrm{C} 152 \mathrm{~S}) \alpha$ & $0.99 \pm 0.067$ & $110 \pm 1.8$ \\
\hline
\end{tabular}

While the substitutions of these cysteine residues did not substantially affect the kinetic parameters of the BcGDH $\gamma \alpha$ complex at room temperature, disulfide bonds often contribute to the stability of the tertiary and/or quaternary structure of proteins. We therefore studied the enzyme activity of the cysteine-substituted mutants at higher temperatures. The wildtype BcGDH $\gamma \alpha$ complex showed maximum activity at $343 \mathrm{~K}$ owing to its high thermal stability, whereas both the $\gamma($ Cys152Ser $) \alpha$ and $\gamma \alpha$ (Cys213Ser) complexes showed maximum activity at approximately 303-313 K (Fig. 5). A substantial decrease in the optimal reaction temperatures of the $\gamma($ Cys152Ser $) \alpha$ and $\gamma \alpha$ (Cys213Ser) complexes suggested an important role of the inter-subunit disulfide bond in maintaining the thermal stability of the wild-type $\operatorname{BcGDH} \gamma \alpha$ complex.

\section{Discussion}

A DALI search (Holm \& Rosenström, 2010; Holm \& Laakso, 2016) revealed many proteins that are structurally similar to the $\alpha$-subunit of $\mathrm{BcGDH} \gamma \alpha$, with high $Z$-scores in the range 23.4-33.0 (Supplementary Table S1). These enzymes are categorized as members of the FAD-containing glucosemethanol-choline oxidoreductase (GMC) family.

Among the identified enzymes in the GMC family, pyranose 2-oxidase (P2Ox) shows the highest similarity to the BcGDH $\alpha$-subunit [Supplementary Figs. S5 $(b)$ and S5 $(d)]$. The structure of cholesterol oxidase (ChOx) is also similar to the structure of the BcGDH $\alpha$-subunit [Supplementary Figs. S5(c) and S5(e)].

The residues responsible for the catalytic reaction have previously been identified in a variety of GMC oxidoreductases. A His/His pair in GOx, fungal FADGDH, pyranose dehydrogenase (PDH) and aryl-alcohol oxidase (AAOx), and a His/Asn pair in cholesterol oxidase (ChOx), cellobiose dehydrogenase $(\mathrm{CDH})$, choline oxidase (COx) and $\mathrm{P} 2 \mathrm{Ox}$ are expected to function as catalytic pairs based on the crystal structures, site-directed mutagenesis, $\mathrm{pH}$-dependence studies or theoretical calculations. The catalytic pair in BcGDH is likely to be His/Asn, represented by His476 and Asn519, and corresponds to His689 and Asn732 in cellobiose dehydrogenase.

The structure of the active site of $\mathrm{BcGDH} \gamma \alpha$ was compared with Phanerochaete chrysosporium cellobiose dehydrogenase (PcCDH) bound to 6-hydroxy-FAD and the inhibitor cellobionolactam (ABL; Hallberg et al., 2003; Supplementary Fig. S6). The His/Asn catalytic pairs (His476/Asn519 in BcGDH $\gamma \alpha$ 
and His689/Asn732 in PcCDH) and the residues recognizing the position of the glucose moiety at the nonreducing end are conserved. Asn688 of PcCDH forms a hydrogen bond to $\mathrm{O} 3$ of $\mathrm{ABL}$, and the carbonyl $\mathrm{O}$ atom of Ser687 in PcCDH contacts $\mathrm{O} 2$ of ABL. The corresponding residues in $\mathrm{BcGDH} \gamma \alpha$ are Asn475 and Asn474, respectively, and would be able to recognize glucose as a substrate. In PcCDH, Glu279 and Arg586 recognize the glucose moiety at the reducing end of cellobiose $[\mathrm{Glc}(\beta 1-4) \mathrm{Glc}]$. The residue corresponding to Arg586 in PcCDH is Ser365 in $\mathrm{BcGDH} \gamma \alpha$, but a residue corresponding to Glu279 in PcCDH has not been identified in $\operatorname{BcGDH} \gamma \alpha$. Although the most favorable substrate of $\mathrm{BcGDH} \gamma \alpha$ is glucose, a large cavity is present in the putative active site of $\mathrm{BcGDH} \gamma \alpha$, as observed in the surface model of
$\mathrm{BcGDH} \gamma \alpha$ superimposed onto the active site of $\mathrm{PcCDH}$ [Supplementary Fig. S6 $(d)$ ], supporting the fact that $\mathrm{BcGDH} \gamma \alpha$ recognizes maltose [ $\mathrm{Glc}(\alpha 1-4) \mathrm{Glc}]$ as a substrate (Yamashita et al., 2013).

In contrast, proteins with a similar structure to the $\gamma$-subunit were not identified in the $D A L I$ search. To date, the $\gamma$-subunit has been considered to be a hitchhiker protein that promotes the secretion of the catalytic subunit to the periplasm. In the bacterial twin-arginine translocation (TAT) pathway, folded proteins are transported across the bacterial cytoplasmic membrane through the recognition of $\mathrm{N}$-terminal signal peptides containing the twin-arginine motif. In some representative twin-arginine signal peptides, $\alpha$-helical regions were predicted using the PSIPRED secondary-structure

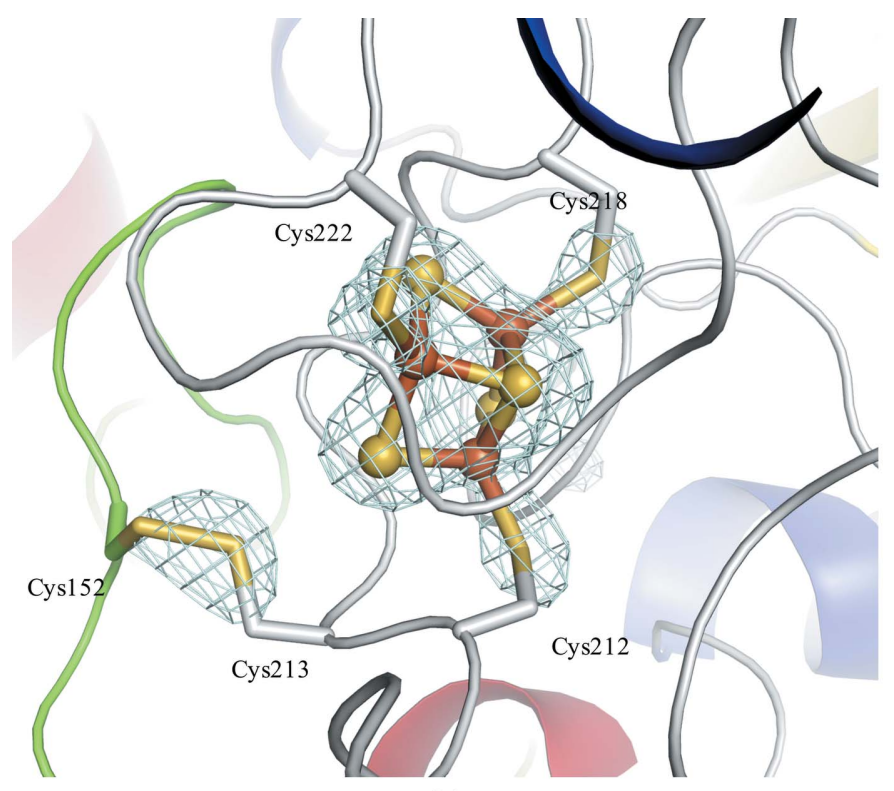

(a)

Cys 218

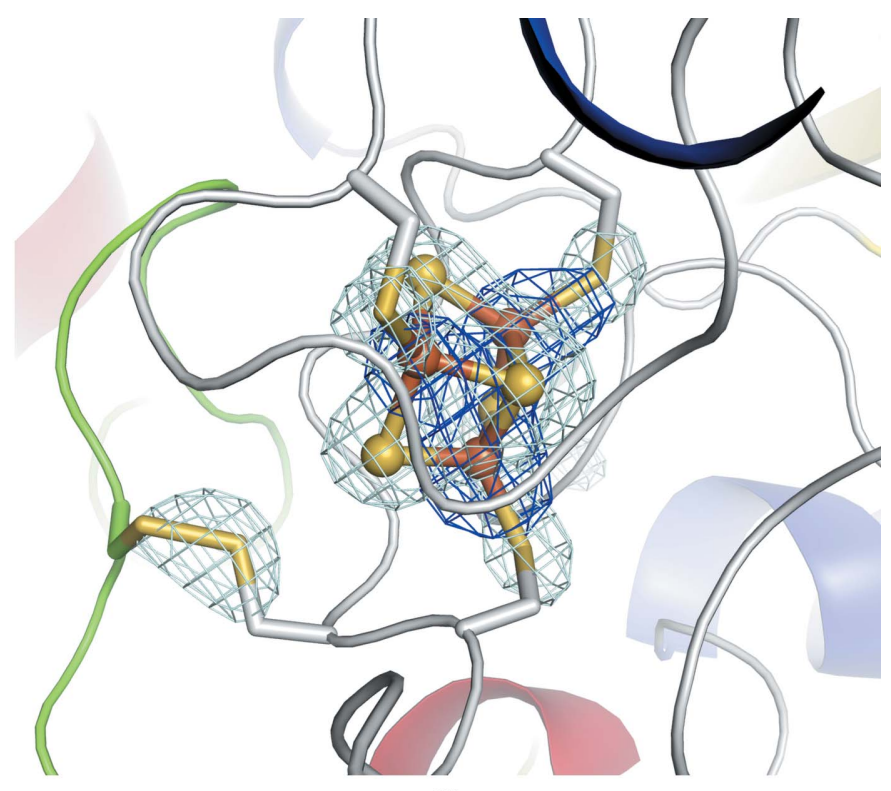

(b)

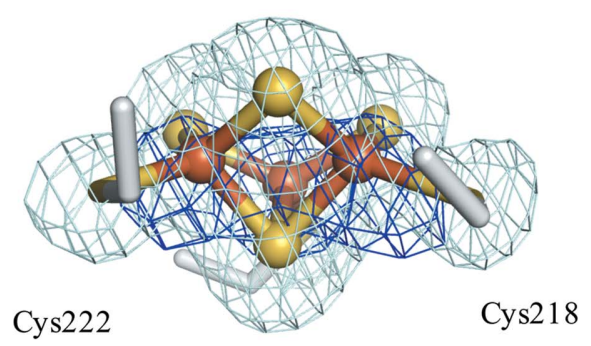

(d)

Figure 3

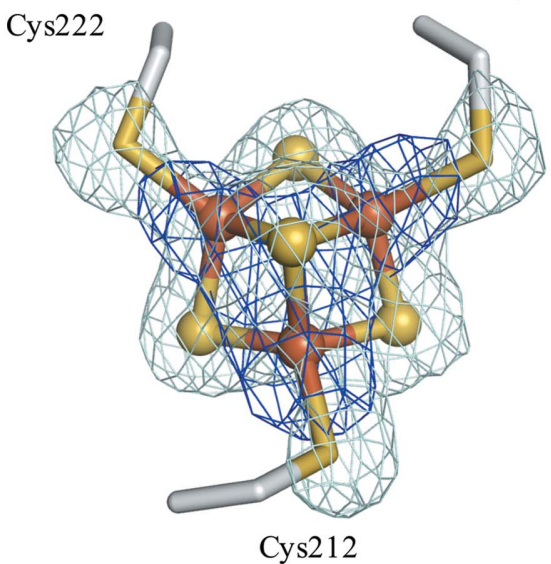

(c)

Cysteine cluster of $\mathrm{BcGDH} \gamma \alpha$. A unique cysteine cluster is formed by one cysteine from the $\gamma$-subunit and four cysteines from the $\alpha$-subunit. Cys152 and Cys213 form a disulfide bond between the $\gamma$ - and $\alpha$-subunits. Cys212, Cys218 and Cys222 are involved in the iron-sulfur cluster (3Fe-4S). The identified positions of the $3 \mathrm{Fe}-4 \mathrm{~S}$ cluster and the disulfide bond between the $\gamma$ - and $\alpha$-subunits are shown. Simulated-annealing OMIT maps of F3S (the 3Fe-4S iron-sulfur cluster) and five cysteine residues (Cys152, Cys212, Cys213, Cys218 and Cys222) in wild-type BcGDH $\gamma \alpha$ contoured at $5 \sigma$ are shown in light blue in $(a)-(d)$. The $2 F_{\mathrm{o}}-F_{\mathrm{c}}$ electron-density maps using the merged data sets of wild-type BcGDH $\gamma \alpha$ collected at wavelengths of 1.0 and $1.74086 \AA$ (after the initial refinement and before including F3S in refinement) are shown in blue and contoured at $5 \sigma$ in $(b),(c)$ and $(d)$. S and Fe atoms are shown in yellow and orange, respectively. The identified $3 \mathrm{Fe}-4 \mathrm{~S}$ cluster consists of three $\mathrm{Fe}$ atoms and four $\mathrm{S}$ atoms represented as spheres. 
prediction method (Palmer et al., 2005). The $\gamma$-subunit of $\mathrm{BcGDH}$ contains the twin-arginine motif in the $\mathrm{N}$-terminal signal peptide and was thought to belong to the Tat protein family. Indeed, the structure of the $\gamma$-subunit of $\mathrm{BcGDH}$ contains five $\alpha$-helices.

The DALI search revealed some proteins with limited homology (Supplementary Table S2 and Fig. S7), including the N-terminal domain (NTD) of Salmonella typhimurium chemotaxis receptor methyltransferase (CheR) in complex with $S$-adenosyl-L-homocysteine (SAH), which had the highest $Z$-score (4.7). These domains of the enzymes were reported to be essential for catalytic activity, although they are located far from the catalytic domain and no role as a hitchhiker protein was reported.

The X-ray structure of $\mathrm{BcGDH} \gamma \alpha$ also revealed the first structure of a hitchhiker protein in complex with the target protein. According to the results of site-directed mutagenesis studies, the formation of disulfide bonds is required to stabilize the catalytic subunit. In other words, the disulfide bond may prevent denaturation of the $3 \mathrm{Fe}-4 \mathrm{~S}$ cluster, thereby maintaining the stability of this enzyme even at temperatures greater than $323 \mathrm{~K}$. Thus, the hitchhiker protein may protect the iron-sulfur cluster until the formation of a complex with the electron-transfer subunit after the catalytic subunit has been secreted into the periplasmic space. The electrontransfer subunit is folded in the periplasmic space and forms a quaternary structure with the catalytic subunit complexed with the hitchhiker protein. However, a mutant catalytic

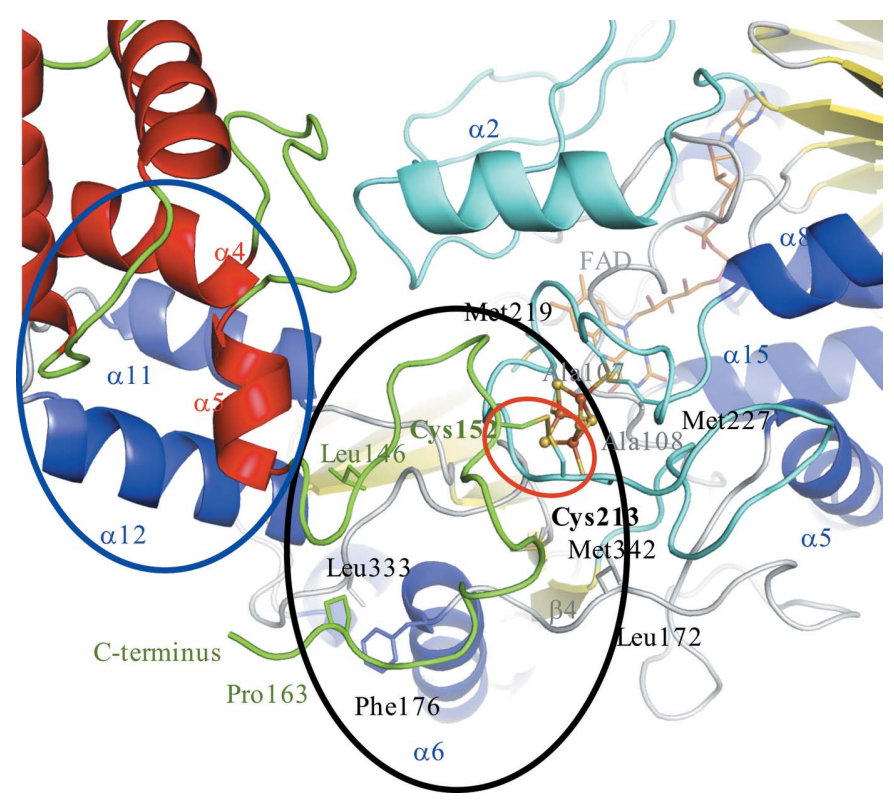

Figure 4

Tightly bound interface between the $\gamma$ - and $\alpha$-subunits. The highly hydrophobic C-terminal tail of the $\gamma$-subunit (colored green) including Cys 152 of the $\gamma$-subunit covers the contact areas like a lid at the interface. The disulfide bond between subunits is indicated by a red circle. One of the long loops of the $\alpha$-subunit including $\alpha 2$ is colored cyan and contacts the C-terminal loop region of the $\gamma$-subunit. The other colors are the same as in Fig. 2. $\alpha 4$ and $\alpha 5$ of the $\gamma$-subunit also make contacts with protruding helices ( $\alpha 11$ and $\alpha 12)$ of the $\alpha$-subunit in the area indicated with a blue circle. subunit or a mutant hitchhiker protein in the enzyme complex was expressed and functional. These results support the lack of a requirement for disulfide bonds in the functional expression and secretion of the complex into the periplasmic space. Indeed, alignments of the primary structures of the catalytic subunits [Supplementary Fig. S8(a)] and hitchhiker proteins [Supplementary Fig. S8(b)] of FAD-dependent dehydrogenase complexes reveal that the cysteine residues are not conserved, indicating that the formation of the disulfide bond is not necessary for a complex to form between catalytic subunits and hitchhiker proteins. Therefore, the hitchhiker protein of FAD-dependent dehydrogenase complexes may mainly interact with and recognize the catalytic subunit throughout these interfacial interactions.

Our previous report revealed the presence of a $3 \mathrm{Fe}-4 \mathrm{~S}$ cluster in BcGDH (Shiota et al., 2016). The X-ray structure of the catalytic subunit clearly indicated the position of the $3 \mathrm{Fe}-4 \mathrm{~S}$ cluster, which is located on the surface of the catalytic subunit. The distance between N5 of FAD and the 3Fe-4S cluster is about $12-13 \AA$, which is an adequate distance for electron transfer. These results support our hypothesis that the $3 \mathrm{Fe}-4 \mathrm{~S}$ cluster functions in the intramolecular electron transfer from FAD and mediates intermolecular electron transfer from the $3 \mathrm{Fe}-4 \mathrm{~S}$ cluster to the electron-transfer subunit. The $3 \mathrm{Fe}-4 \mathrm{~S}$ cluster is responsible for electron transfer and interacts with the multi-heme $c$ electron-transfer subunit.

As seen in the electron-density map, a covalent bond might form between C8M of FAD and His105 in the $\alpha$-subunit of BcGDH [Fig. 2(b)]. The position of His105 is conserved in the GMC oxidoreducatase family, such as in P2Oxs (Bannwarth et al., 2004; Halada et al., 2003; Hallberg et al., 2004; Hassan et al., 2013; Spadiut et al., 2010; Tan et al., 2013), COxs (Quaye et al., 2008) and fumarate reductases B (Iverson et al., 1999, 2003;

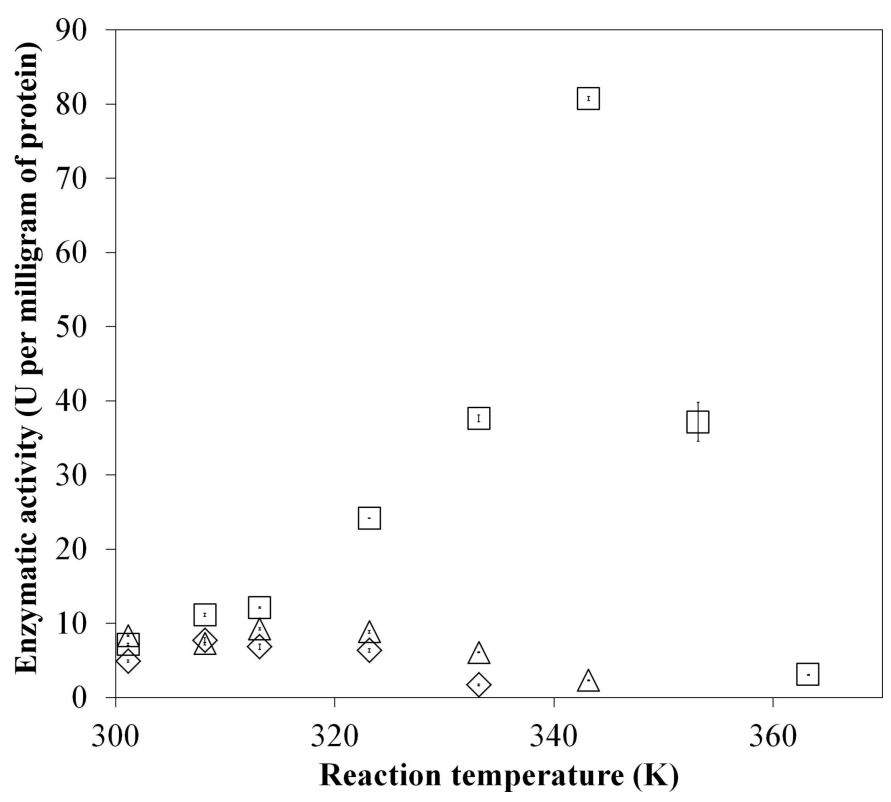

Figure 5

The effect of temperature on the enzymatic activities of wild-type and mutant $\mathrm{BcGDH} \gamma \alpha$. Dye-mediated glucose dehydrogenase activities of wild-type (open squares), $\gamma$ (Cys152Ser) $\alpha$ (open triangles) and $\gamma \alpha$ (Cys213Ser) (open diamonds) BcGDH at each temperature are shown. 


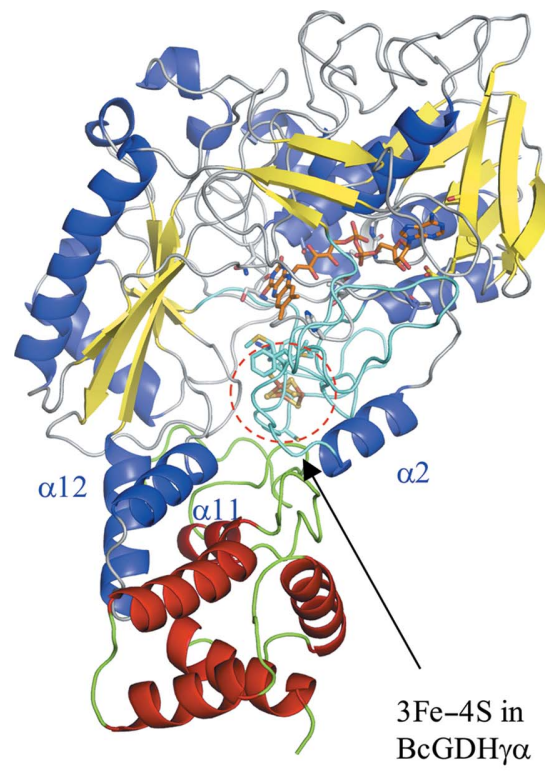

(a)

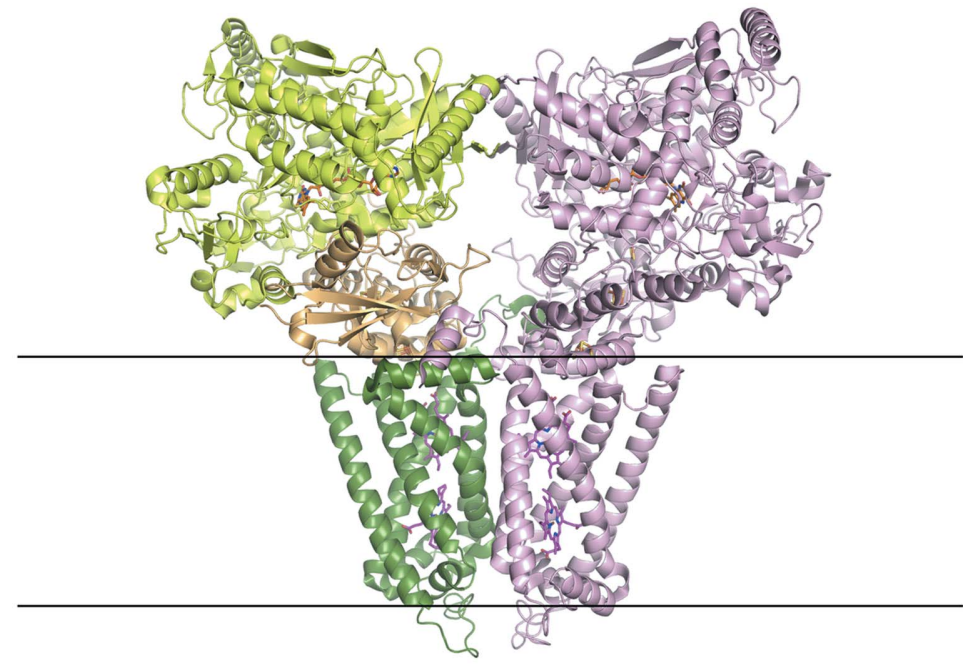

(b)

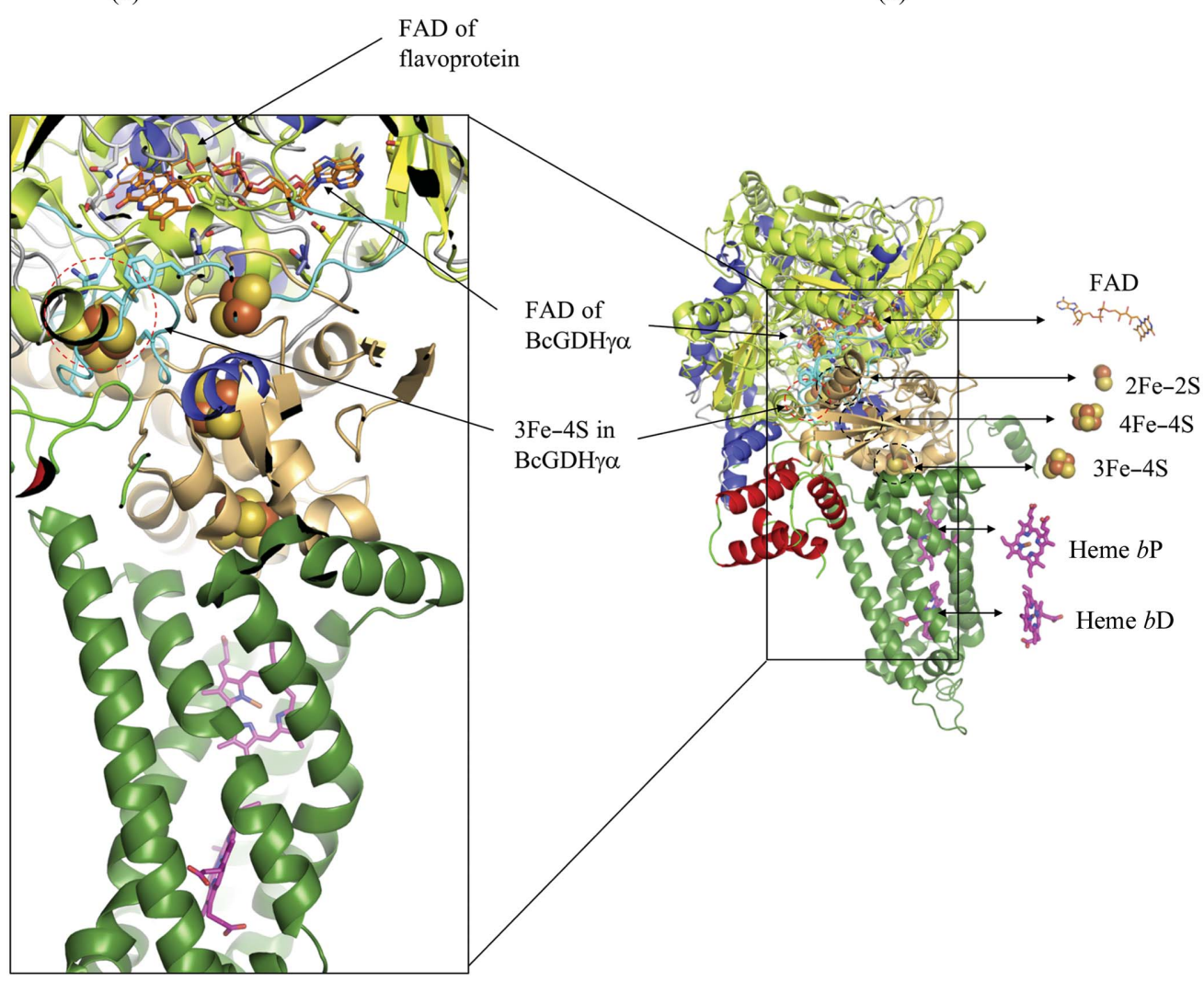

(c)

Figure 6

The structures of $(a) \mathrm{BcGDH} \gamma \alpha,(b) W$. succinogenes membrane-bound fumarate reductase (PDB entry 2bs2; Madej et al., 2006) and the flavoprotein shown in light green in $(b)$ were superimposed onto the structure of BcGDH $\gamma \alpha$ in $(c)$. The colors of BcGDH $\gamma \alpha$ are the same as in Fig. 2. The 3Fe-4S cluster is indicated by a red dotted circle. Soluble S. putrefaciens fumarate reductase was first superimposed onto the structure of the $\alpha$-subunit of $\mathrm{BcGDH} \gamma \alpha$ with 3.0 r.m.s.d., and the membrane-bound fumarate reductase from W. succinogenes was superimposed onto the structure of soluble fumarate reductase from $S$. putrefaciens (with 1.8 r.m.s.d.). Consequently, the structure of the membrane-bound fumarate reductase was directly compared with the structure of $\mathrm{BcGDH} \gamma \alpha$ by removing the structure of the soluble fumarate reductase. (b) The two-molecule form of fumarate reductase is shown in the crystal structure (Lancaster et al., 1999). A molecule of fumarate reductase is represented in light pink (right). In the other molecule, subunit A (flavoprotein containing FAD covalently bound to His 43 ), subunit B (iron-sulfur protein including $2 \mathrm{Fe}-2 \mathrm{~S}$, $4 \mathrm{Fe}-4 \mathrm{~S}$ and $3 \mathrm{Fe}-4 \mathrm{~S}$ clusters) and subunit $\mathrm{C}$ (transmembrane-spanning protein including diheme cytochrome $b$ molecules) are colored light green, light orange and dark green, respectively. The bound FAD molecules in the structures are shown as orange stick models. The bound FAD and heme $b$ molecules are shown as orange lines and magenta stick models, respectively. The iron-sulfur clusters are shown as sphere models. The other molecule of fumarate reductase colored light pink was deleted in $(c)$ to clarify the positions of FAD, iron-sulfur clusters and heme $b$ molecules, which are related to electron transfer. $b \mathrm{P}$ is the proximal heme and $b \mathrm{D}$ is the distal heme. 
Lancaster et al., 2001; Madej et al., 2006), and this residue forms covalent bonds with FAD.

Next, we attempted to predict the position of the electrontransfer subunit by comparing of the structures of $\mathrm{CDH}$ and fumarate reductase, considering the previously elucidated intramolecular and intermolecular electron-transfer pathways (Shiota et al., 2016; Yamashita et al., 2018), as well as the nature of the electron-transfer subunit (Okuda-Shimazaki et al., 2018).

In Supplementary Fig. S9, the structure of the catalytic domain of $\mathrm{CDH}$ (closed state) was superimposed onto the structure of the $\alpha$-subunit of BcGDH $\gamma \alpha$ [Supplementary Fig. $\mathrm{S} 9(d)]$. The $3 \mathrm{Fe}-4 \mathrm{~S}$ cluster between the $\gamma$ - and $\alpha$-subunits of $\mathrm{BcGDH}$ (red dotted circle) is located on the left side, which is the opposite side to the heme $b$-type (magenta stick) electrontransfer domain of $\mathrm{CDH}$. Considering that the primary electron acceptor of FAD is the $3 \mathrm{Fe}-4 \mathrm{~S}$ cluster, and that in the next step intermolecular electron transfer occurs between the $3 \mathrm{Fe}-4 \mathrm{~S}$ cluster and the electron-transfer subunit, the position of the electron-transfer subunit of BcGDH would be opposite to that of the heme $b$-type electron-transfer domain of $\mathrm{CDH}$ in the closed state.

Soluble flavocytochrome $c$ fumarate reductase from Shewanella putrefaciens is a periplasmic tetraheme flavocytochrome $c$ that consists of an $\mathrm{N}$-terminal tetraheme cytochrome $c$ domain and a catalytic region that contains the three $\mathrm{C}$-terminal domains. The $\mathrm{N}$-terminal domain containing the tetraheme moiety is connected by an $\alpha$-helical linker to the FAD-binding catalytic domain with noncovalently bound FAD. Membrane-bound diheme-containing quinol:fumarate reductase (QFR) from Wolinella succinogenes is composed of three subunits (A, B and C), in which subunit A contains the catalytic site of fumarate reduction and an FAD covalently bound to His43. Subunit B contains three iron-sulfur clusters and subunit $\mathrm{C}$ is a diheme cytochrome $b$ (Lancaster et al., 2001; Madej et al., 2006).

Focusing on the homology between the soluble flavocytochrome $c$ fumarate reductase from $S$. putrefaciens and the membrane-bound fumarate reductase from $W$. succinogenes, as well as the homology between soluble flavocytochrome $c$ fumarate reductase from $S$. putrefaciens and the $\alpha$-subunit of $\mathrm{BcGDH}$, the structure of membrane-bound fumarate reductase from $W$. succinogenes was superimposed onto the structure of the $\alpha$-subunit of BcGDH $\gamma \alpha$ (Fig. 6). As shown in the membrane-bound fumarate reductase, the electron is transferred from FAD to the transmembrane protein containing heme $b \mathrm{P}$ and heme $b \mathrm{D}$ via $2 \mathrm{Fe}-2 \mathrm{~S}, 4 \mathrm{Fe}-\mathrm{S}$ and $3 \mathrm{Fe}-4 \mathrm{~S}$ clusters. Interestingly, the positions of FAD and the first $\mathrm{Fe}-\mathrm{S}$ cluster, $2 \mathrm{Fe}-2 \mathrm{~S}$, of fumarate reductase, with a distance of $12.3 \AA$, are comparable to those of $\mathrm{FAD}$ and the $3 \mathrm{Fe}-4 \mathrm{~S}$ cluster of BcGDH $\gamma \alpha$, with a distance of around 12-13 $\AA$. The distances between $\mathrm{Fe}-\mathrm{S}$ clusters are $11.0 \AA(2 \mathrm{Fe}-2 \mathrm{~S}$ and $4 \mathrm{Fe}-4 \mathrm{~S})$ and $9.1 \AA(4 \mathrm{Fe}-4 \mathrm{~S}$ and $3 \mathrm{Fe}-4 \mathrm{~S})$, whereas the distances from the $\mathrm{Fe}-\mathrm{S}$ clusters to the heme domains are $17.6 \AA(3 \mathrm{Fe}-4 \mathrm{~S}$ and cytochrome $b \mathrm{P}$ ) and $15.6 \AA$ (cytochrome $b \mathrm{P}$ and cytochrome $b \mathrm{D})$. Although membrane-bound fumarate reductase contains three $\mathrm{Fe}-\mathrm{S}$ clusters and two heme domains, the $\beta$-subunit of
BcGDH contains three heme $c$ moieties in its electron-transfer subunit. The intact BcGDH complex including the membranebound $\beta$-subunit containing three heme $c$ moieties may form a similar overall structure to fumarate reductases for effective electron transfer. Interestingly, our previous study on the $\beta$-subunit suggested that the electron from the $\mathrm{Fe}-\mathrm{S}$ cluster is initially transferred to the third heme in the $\beta$-subunit (the $\mathrm{C}$-terminal heme domain of the $\beta$-subunit), is then transferred to the second heme, is further transferred to the first heme (the $\mathrm{N}$-terminal heme domain of the $\beta$-subunit) and is finally transferred to an external artificial electron acceptor. However, when the intact BcGDH is immobilized on the electrode, the electron is transferred from the third heme to the second heme, and is then directly transferred to the electrode. The third heme of $\mathrm{BcGDH} \gamma \alpha \beta$ may correspond to the position between $4 \mathrm{Fe}-4 \mathrm{~S}$ and $3 \mathrm{Fe}-4 \mathrm{~S}$ of fumarate reductase, the second heme corresponds to heme $b \mathrm{P}$ and the first heme corresponds to heme $b \mathrm{D}$.

In conclusion, this study reports the first X-ray structure of a representative DET-type FAD-dependent dehydrogenase complex: the BcGDH catalytic subunit complexed with a hitchhiker protein. The structure of $\mathrm{BcGDH} \gamma \alpha$ revealed a conserved GMC oxidoreductase-type scaffold and a His/Asn catalytic pair, with a unique structure of the $3 \mathrm{Fe}-4 \mathrm{~S}$ cluster, which serves as the electron acceptor of FAD and simultaneously serves as the electron donor for electron transfer of the multiheme $c$ subunit. These findings will be essential for improving our understanding of intramolecular and intermolecular electron transfer by DET-type FAD-dependent dehydrogenase complexes, as well as for engineering DETtype enzymes for the development of future bioelectrochemical devices.

\section{Related literature}

The following references relate to PDB entries that are mentioned in the supporting information to this article: Batra et al. (2016), Djordjevic \& Stock (1997, 1998), Golden et al. (2014), Leys et al. (1999), Liu et al. (2015), Mugo et al. (2013), Pitsawong et al. (2010), Salvi et al. (2014), Tan et al. (2015), Wohlfahrt et al. (1999), Yoshida et al. (2015) and Zhang et al. (2014).

\section{Acknowledgements}

The authors would like to thank Mr Kentaro Hiraka, Department of Biotechnology and Life Science, Graduate School of Engineering, Tokyo University of Agriculture and Technology for his technical assistance. The authors are also grateful to Dr Alexander Wlodawer, Senior Investigator, Macromolecular Crystallography Laboratory, Center for Cancer Research, National Cancer Institute, National Institutes of Health, USA for his kind efforts in the English language review of the manuscript. This study was performed after obtaining the approval of the Photon Factory Program Advisory Committee (Proposal Nos. 2015G534 and 2017G610). 


\section{Funding information}

This study was partially supported by JSPS KAKENHI grant JP16H04175 to KS.

\section{References}

Adams, P. D., Afonine, P. V., Bunkóczi, G., Chen, V. B., Davis, I. W., Echols, N., Headd, J. J., Hung, L.-W., Kapral, G. J., GrosseKunstleve, R. W., McCoy, A. J., Moriarty, N. W., Oeffner, R., Read, R. J., Richardson, D. C., Richardson, J. S., Terwilliger, T. C. \& Zwart, P. H. (2010). Acta Cryst. D66, 213-221.

Afonine, P. V., Grosse-Kunstleve, R. W., Echols, N., Headd, J. J., Moriarty, N. W., Mustyakimov, M., Terwilliger, T. C., Urzhumtsev, A., Zwart, P. H. \& Adams, P. D. (2012). Acta Cryst. D68, 352-367.

Ameyama, M., Shinagawa, E., Matsushita, K. \& Adachi, O. (1981). J. Bacteriol. 145, 814-823.

Bannwarth, M., Bastian, S., Heckmann-Pohl, D., Giffhorn, F. \& Schulz, G. E. (2004). Biochemistry, 43, 11683-11690.

Batra, M., Sharma, R., Malik, A., Dhindwal, S., Kumar, P. \& Tomar, S. (2016). J. Struct. Biol. 196, 364-374.

Djordjevic, S. \& Stock, A. M. (1997). Structure, 5, 545-558.

Djordjevic, S. \& Stock, A. M. (1998). Nature Struct. Biol. 5, 446-450.

Emsley, P., Lohkamp, B., Scott, W. G. \& Cowtan, K. (2010). Acta Cryst. D66, 486-501.

Golden, E., Karton, A. \& Vrielink, A. (2014). Acta Cryst. D70, 31553166.

Halada, P., Leitner, C., Sedmera, P., Haltrich, D. \& Volc, J. (2003). Anal. Biochem. 314, 235-242.

Hallberg, B. M., Henriksson, G., Pettersson, G., Vasella, A. \& Divne, C. (2003). J. Biol. Chem. 278, 7160-7166.

Hallberg, B. M, Leitner, C., Haltrich, D. \& Divne, C. (2004). J. Mol. Biol. 341, 781-796.

Hassan, N., Tan, T. C., Spadiut, O., Pisanelli, I., Fusco, L., Haltrich, D., Peterbauer, C. K. \& Divne, C. (2013). FEBS Open Bio, 3, 496-504.

Holm, L. \& Laakso, L. M. (2016). Nucleic Acids Res. 44, W351-W355.

Holm, L. \& Rosenström, P. (2010). Nucleic Acids Res. 38, W545W549.

Inose, K., Fujikawa, M., Yamazaki, T., Kojima, K. \& Sode, K. (2003). Biochim. Biophys. Acta, 1645, 133-138.

Iverson, T. M., Luna-Chavez, C., Cecchini, G. \& Rees, D. C. (1999). Science, 284, 1961-1966.

Iverson, T. M., Luna-Chavez, C., Croal, L. R., Cecchini, G. \& Rees, D. C. (2002). J. Biol. Chem. 277, 16124-16130.

Kataoka, N., Matsutani, M., Yakushi, T. \& Matsushita, K. (2015). Appl. Environ. Microbiol. 81, 3552-3560.

Kawai, S., Goda-Tsutsumi, M., Yakushi, T., Kano, K. \& Matsushita, K. (2013). Appl. Environ. Microbiol. 79, 1654-1660.

Lancaster, C. R., Gross, R. \& Simon, J. (2001). Eur. J. Biochem. 268, 1820-1827.

Lancaster, C. R., Kröger, A., Auer, M. \& Michel, H. (1999). Nature (London), 402, 377-385.

Laskowski, R. A., MacArthur, M. W., Moss, D. S. \& Thornton, J. M. (1993). J. Appl. Cryst. 26, 283-291.

Laskowski, R. A., MacArthur, M. W. \& Thornton, J. M. (2001). International Tables for Crystallography, Vol. F, edited by M. G. Rossmann \& E. Arnold, pp. 722-725. Dordrecht: Kluwer Academic Publishers.

Leys, D., Tsapin, A. S., Nealson, K. H., Meyer, T. E., Cusanovich, M. A. \& Van Beeumen, J. J. (1999). Nature Struct. Biol. 6, 11131117.

Liu, R. J., Long, T., Zhou, M., Zhou, X. L. \& Wang, E. D. (2015). Nucleic Acids Res. 43, 7489-7503.

Madej, M. G., Nasiri, H. R., Hilgendorff, N. S., Schwalbe, H. \& Lancaster, C. R. (2006). EMBO J. 25, 4963-4970.
Mugo, A. N., Kobayashi, J., Yamasaki, T., Mikami, B., Ohnishi, K., Yoshikane, Y. \& Yagi, T. (2013). Biochim. Biophys. Acta, 1834, 953963.

Murshudov, G. N., Skubák, P., Lebedev, A. A., Pannu, N. S., Steiner, R. A., Nicholls, R. A., Winn, M. D., Long, F. \& Vagin, A. A. (2011). Acta Cryst. D67, 355-367.

Okuda-Shimazaki, J., Loew, N., Hirose, N., Kojima, K., Mori, K., Tsugawa, W. \& Sode, K. (2018). Electrochim. Acta, 277, 276-286.

Otwinowski, Z. \& Minor, W. (1997). Methods Enzymol. 276, 307326.

Palmer, T., Sargent, F. \& Berks, B. C. (2005). Trends Microbiol. 13, 175-180.

Pitsawong, W., Sucharitakul, J., Prongjit, M., Tan, T. C., Spadiut, O., Haltrich, D., Divne, C. \& Chaiyen, P. (2010). J. Biol. Chem. 285, 9697-9705.

Quaye, O., Lountos, G. T., Fan, F., Orville, A. M. \& Gadda, G. (2008). Biochemistry, 47, 243-256.

Salvi, F., Wang, Y.-F., Weber, I. T. \& Gadda, G. (2014). Acta Cryst. D70, 405-413.

Shinagawa, E., Matsushita, K., Adachi, O. \& Ameyama, M. (1981). Agric. Biol. Chem. 45, 1079-1085.

Shiota, M., Yamazaki, T., Yoshimatsu, K., Kojima, K., Tsugawa, W., Ferri, S. \& Sode, K. (2016). Bioelectrochemistry, 112, 178-183.

Sode, K., Tsugawa, W., Yamazaki, T., Watanabe, M., Ogasawara, N. \& Tanaka, M. (1996). Enzyme Microb. Technol. 19, 82-85.

Sode, K., Yamazaki, T., Lee, I., Hanashi, T. \& Tsugawa, W. (2016). Biosens. Bioelectron. 76, 20-28.

Spadiut, O., Tan, T. C., Pisanelli, I., Haltrich, D. \& Divne, C. (2010). FEBS J. 277, 2892-2909.

Studier, F. W. (2005). Protein Expr. Purif. 41, 207-234.

Tan, T. C., Kracher, D., Gandini, R., Sygmund, C., Kittl, R., Haltrich, D., Hällberg, B. M., Ludwig, R. \& Divne, C. (2015). Nature Commun. 6, 7542 .

Tan, T. C., Spadiut, O., Wongnate, T., Sucharitakul, J., Krondorfer, I., Sygmund, C., Haltrich, D., Chaiyen, P., Peterbauer, C. K. \& Divne, C. (2013). PLoS One, 8, e53567.

Terwilliger, T. (2004). J. Synchrotron Rad. 11, 49-52.

Toyama, H., Soemphol, W., Moonmangmee, D., Adachi, O. \& Matsushita, K. (2005). Biosci. Biotechnol. Biochem. 69, 11201129.

Tsuya, T., Ferri, S., Fujikawa, M., Yamaoka, H. \& Sode, K. (2006). J. Biotechnol. 123, 127-136.

Winn, M. D., Ballard, C. C., Cowtan, K. D., Dodson, E. J., Emsley, P., Evans, P. R., Keegan, R. M., Krissinel, E. B., Leslie, A. G. W., McCoy, A., McNicholas, S. J., Murshudov, G. N., Pannu, N. S., Potterton, E. A., Powell, H. R., Read, R. J., Vagin, A. \& Wilson, K. S. (2011). Acta Cryst. D67, 235-242.

Wohlfahrt, G., Witt, S., Hendle, J., Schomburg, D., Kalisz, H. M. \& Hecht, H.-J. (1999). Acta Cryst. D55, 969-977.

Yamaoka, H., Ferri, S., Fujikawa, M. \& Sode, K. (2004). Biotechnol. Lett. 26, 1757-1761.

Yamaoka, H. \& Sode, K. (2007). Open Biotechnol. J. 1, 26-30.

Yamaoka, H., Yamashita, Y., Ferri, S. \& Sode, K. (2008). Biotechnol. Lett. 30, 1967-1972.

Yamashita, Y., Ferri, S., Huynh, M. L., Shimizu, H., Yamaoka, H. \& Sode, K. (2013). Enzyme Microb. Technol. 52, 123-128.

Yamashita, Y., Lee, I., Loew, N. \& Sode, K. (2018). Curr. Opin. Electrochem. 12, 92-100.

Yamazaki, T., Tsugawa, W. \& Sode, K. (1999). Appl. Biochem. Biotechnol. 77, 325-336.

Yoshida, H., Sakai, G., Mori, K., Kojima, K., Kamitori, S. \& Sode, K. (2015). Sci. Rep. 5, 13498.

Zhang, Z., Wu, J., Lin, W., Wang, J., Yan, H., Zhao, W., Ma, J., Ding, J., Zhang, P. \& Zhao, G.-P. (2014). J. Biol. Chem. 289, 2796627978. 